\title{
Optimization of Styrene Production Process using Divided Wall Column
}

\author{
Tanmay Kakade ${ }^{1}$, Niraj Topare ${ }^{2}$ \\ ${ }^{1,2}$ Maharashtra Institute of Technology, Department of Petrochemical Engineering, Pune, Maharashtra 411038, India
}

\begin{abstract}
Styrene which is known to be one of the most expensive monomers is conventionally produced by dehydrogenation of ethyl benzene which generates a mixture of benzene, toluene and styrene. The separation of this tertiary mixture is done through two column distillation by first removing benzene from the first column and then separating toluene from styrene in the second column. The conventional distillation system consumes high-energy and yields low purity of toluene.Therefore to overcome these drawbacks we have proposed the use of a Divided Wall Column. In this work, we have designed a Divided wall column (DWC) for styrene production and compared it with the conventional method. By using DWC arrangement we achieved less energy consumption and higher purity of products compared to the conventional method. The simulation of the DWC was done using four column arrangement in Aspen Plus software. The results show that, energy consumed when divided wall column was considered is far less as compared to conventional system used in industry. With the application of DWC column, higher purity toluene was obtained (76.15\% to $96.00 \%)$ and for styrene purity increased from $99.67 \%$ to $99.99 \%$.
\end{abstract}

Keywords: conventional distillation column, Divided wall column, Aspen Plus, high purity, energy saving

\section{Introduction}

Styrene is one of the most important monomers in modern petrochemical industry. Approximately 20 million tons of styrene is produced annually across the globe. The styrene production process was developed in the 1930s independently and simultaneously by BASF in Germany and by Dow Chemical in the USA. It is used for the production of many different polymeric materials, the most important being polystyrene, styrene-acrylonitrile and acrylonitrilebutadiene-styrene (ABS). Another important application is in styrene-butadiene latex. The most important ways of production of styrene are the catalytic dehydrogenation of ethyl benzene (EB) and the oxidation of EB to ethyl benzene hydro-peroxide. Ethyl-benzene (EB) de-hydrogenation is the main route of styrene production, being among the most popular and important catalyzed processes. Investigations in this field have consolidated this process which uses most of the raw material for styrene production with low levels of byproducts. Although the by products are just a small percentage of the raw material, the generated compounds are valuable and it is important they have enough degree of purity to be commercialized. The most common technique to obtain each distillation equipment that increases the process energy efficiency has been modest, once the main interest is on stage of the final stream components is distillation, which allows the separation of styrene, toluene and benzene as the main reaction products. Distillations is still the best method and option available for the separation of compounds with no azeotropes formation and have a wide temperature range for the treatment of output stream coming from the reactor in order to separate the products from the product stream. The implementation of modern distillation equipment that increases the process energy efficiency has been modest, once the main interest is on stage optimization of catalyzed system reaction. Divided Wall Column is among this type of distillation system, consisting of a single distillation column with bidirectional flow. Instead of using two separate vessels, a practical implementation is to use a single vessel with an internal wall that separates the feed and side stream sides of the vessel. This is what we call as a Divided-Wall Column (DWC). The main advantages of this system are the increased the purity of the styrene/toluene and moreover reduction of the energy consumption compared to the conventional distillation system, resulting in lower costs of equipment construction and less energy consumption [1]. As a result, an economical system is obtained, which is superior the conventional system in the above-mentioned ways. For this reason, it is important. For this reason, it is important to develop distillation equipment such as Divided Wall Column for benzene-toluene-styrene system, as this type of technology enhances considerably the purity of product associated to conventional separation methods of such system.

\section{Conventional Method}

The Conventional method for styrene production is the dehydrogenation of ethylbenzene, widely used for the industrial production of styrene. This method was first devised in mid 1930s. The production of styrene increased dramatically during the 1940 s, when it gained its popularity as a feedstock for synthetic rubber. The conventional design of the separation section of a benzene-toluene-styrene system consists of two continuous columns from which benzene is extracted first, as it is the most volatile compound. Then, the styrene stream is purified in the second column through removal of toluene. In this case, benzene forms an independent stream after leaving the first column. The remaining product continues through the second column, where the toluene stream leaves the process with a good purity from the top while styrene is collected from the bottom of the column. Even though, the system is pretty much simple to understand but making a system giving more purity is need of hour in this technologically advanced world. 


\section{International Journal of Science and Research (IJSR) \\ ISSN (Online): 2319-7064}

Index Copernicus Value (2015): 78.96 | Impact Factor (2015): 6.391

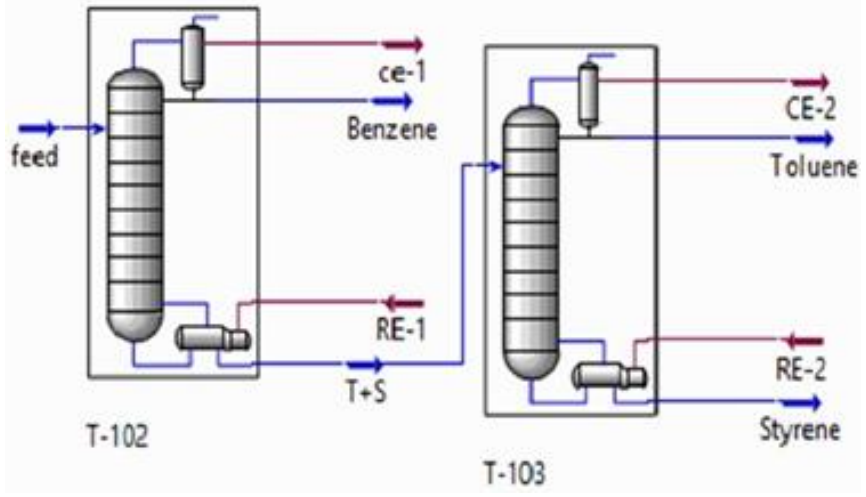

Figure 1: Conventional distillation system for styrene production

The stream from a reactor used in styrene production is analysed in order to compare the energy economy produced with the implementation of a Divided Wall Column and a conventional system. The conventional ethyl benzene dehydrogenation process is the most used production process of styrene in industrial scale. The stream composition is $85 \%$ styrene, $10 \%$ benzene and $5 \%$ toluene on mass basis, with a total mole flow rate of $9667 \mathrm{kgmole} / \mathrm{h} \mathrm{[2].} \mathrm{This} \mathrm{stream} \mathrm{was}$ evaluated by the Aspen Hysys software; a professional simulation program for chemical processes. The Aspen Hysys software simulated the conventional separation system to determine components with adequate purity for commercialization. From the separation process model the number of stages used for separation as well as the energy consumption of the system was obtained. This way, 24 stages were required by the distillation column for benzene separation, while, for toluene and styrene removal, 40 stages were required. The total energy used in the conventional process was $2199.49 \mathrm{~kW}$, which compares well with the results reported by other authors for the same separation process.

\section{Divided Wall Column}

In the case of a divided wall column, instead of two columns (as seen conventionally), there is only one column divided into two parts. The first part of the column is the prefractionator, which helps in separating the stream into two parts, for example containing Benzene with Toluene, and the other Styrene with Toluene. These two streams are led into the main distillation, wherein they are purified, separated and extracted. The partition wall in the middle section of the column separates the main column from the side column [3]. These columns being thermally coupled, the multicomponent feed enters the main column, where a cut between high and low boiling components takes place. This ensures that, taking the example mentioned above, neither low boiling components of Benzene can pass to the bottom part of the side column, nor high boiling components of Styrene can pass through the upper part of the side column, ensuring no contamination of middle boiling fraction of Toluene. This method is a cost, and energy efficient alternative to the conventional two column system, due to having one column and one re-boiler and one condenser less [4].From modelling the divided wall column (DWC) system to obtain the products at the same conditions, as those obtained from conventional systems, we obtain higher purity levels of Toluene and Styrene, which is one of the main advantages of the DWC. This way the DWC required energy of $1927.09 \mathrm{KW}$ which is around $14.5 \%$ less than the conventional system.

\section{Simulation Model}

The simulation of DWC was done using aspen plus software. The Radfrac column available in the component library of aspen plus was used. The steady state simulation of aspen plus was done using Four 'fictious' columns: 'two absorbers', 'rectifier', 'stripper' were used to represent the DWC column. In reality there is only one column, but in aspen plus four columns represent the single DWC column [5]. In this simulation stream 'feed' is fed into the reactor. Benzene, Toluene and Styrene are represented by streams 'Benzene', 'Toluene 'and 'Styrene' respectively.

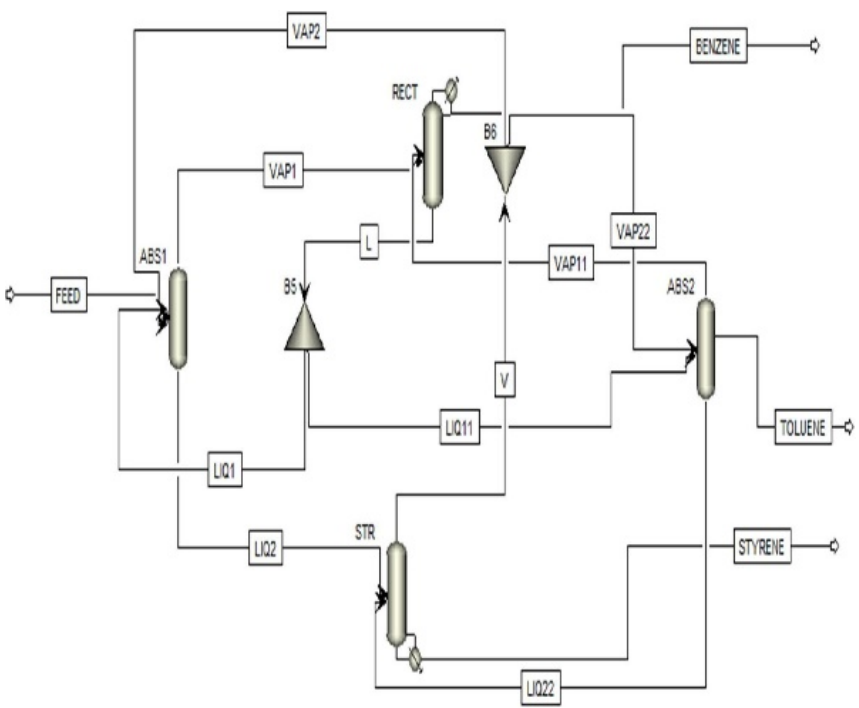

Figure 2: Divided Wall Column for Styrene Production simulated in Aspen plus.

A liquid splitter and vapor splitter are present represented by 'B5' and 'B6' respectively. Pressure is maintained constant through the simulation [6]. The variables to be initialized for simulation were: number of trays in the column, feed stage, vapor/liquid split ratio. Before exporting the file in aspen dynamics a number of important changes were to be made for the dynamic simulation of the model.

\section{Mathematical Model}

The mathematical model for the simulation of conventional method and divided wall column was done using mathematical iterative methods. Two iterative methods were used. Firstly the Fenske Underwood Gilliland equation method was used followed by a rigorous method. The algorithm for the Fenske Underwood method is shown in the Figure 3 [7]. The minimum numbers of trays were calculated using the Fenske equation. The dew point and the bubble point temperature were calculated by assuming equilibrium between the liquid and vapour at each tray. To finally design the column it is necessary to calculate the pressure, temperature, composition, stream flows and rates of heat and

\section{Volume 6 Issue 4, April 2017 www.ijsr.net}




\section{International Journal of Science and Research (IJSR) \\ ISSN (Online): 2319-7064}

Index Copernicus Value (2015): 78.96 | Impact Factor (2015): 6.391

mass transfer in each tray through energy and mass balances. These balances require us to solve a lot many equations to calculate the minimum number of trays required by the distillation process. The columns in the aspen plus software are simulated till the required results are obtained which are in comparison with the results obtained from the Fenske Underwood Gilliland equation.

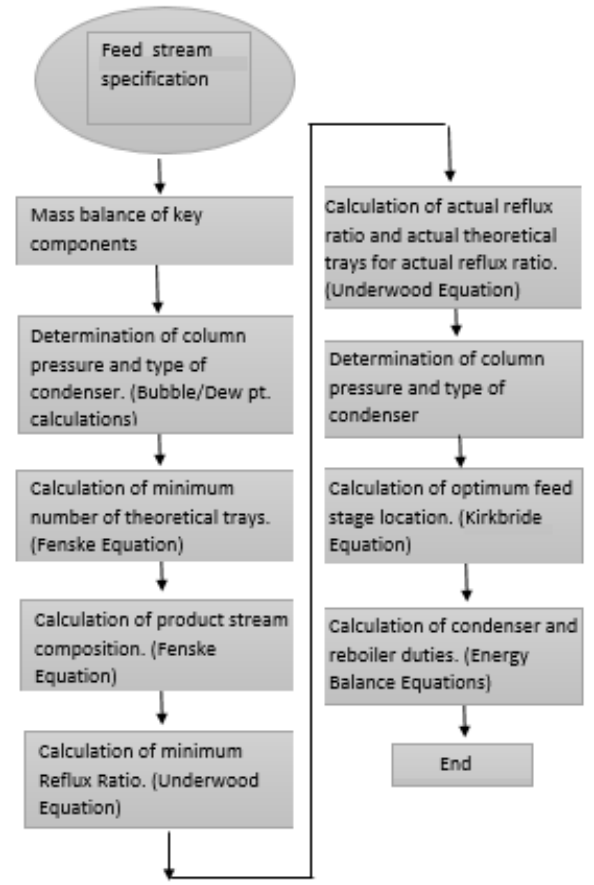

Figure 3: Algorithm of the Fenske Underwood Gilliland Equation

\section{Data Analysis}

Based on the simulation performed, the divided wall column system is analysed and compared with the conventional distillation system. The system structure is presented by the four graphs (Fig.4, Fig.5, Fig6, Fig.7, Fig.8).

1)In Figure 4, graph (Purity vs reflux ratio) we compare the purity of each product against the variation of reflux ratio. As seen from the graph(Fig.3) we observe that as the reflux ratio increases the purity of all 3 components decreases . We get higher purity of all components when simulated at reflux ratio equals to 1.97 .

2)In Figure 5, graph (Purity vs Distillate rate) compares the purity of each product against the variation of distillate rate. From the graph, we observe that purity of benzene, toluene and styrene is maximum for distillate rate equals to $739.9 \mathrm{kgmol} / \mathrm{h}$. Generally as we decrease the distillate rate, the purity of styrene remains unchanged but the purity of benzene and toluene increases considerably.

3) In the Figure 6, graph(Mole fraction vs Stage number) in the rectifier section as the Stage number increases the mole fraction of styrene remains constant (approximately zero), toluene increases gradually up to stage 11, increases exponentially upto stage 21 and then becomes gradual till stage 24 and that of benzene decreases up to stage 13 ,then decreases exponentially up to stage 21 and then remains constant. General observation is the Stage number does not have not much effect on the mole fractions of Benzene and toluene.

4)In the Figure7, graph (Mole fraction vs Stage number) in the stripper section as the Stage number increases the mole fraction of styrene gradually increases till stage 46, that of toluene gradually decreases and that of benzene remains approximately zero.

5)In the Figure 8, graph (Mole fraction vs Stage number) in the section from which toluene is collected (ABS 2) as the Stage number increases the mole fraction of toluene decreases (96\%-93\%) till stage 24, that of styrene gradually increases $(0.1 \%-70 \%)$ and that of benzene (approx. 0) remains approximately constant.



Figure 4: Purity vs Reflux Ratio

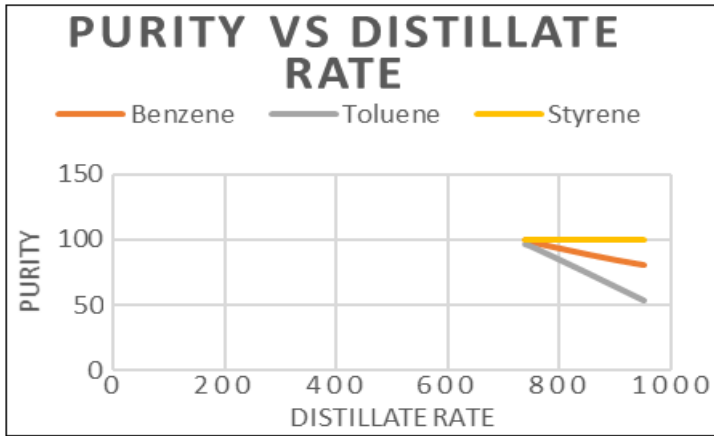

Figure 5: Purity vs Distillate Rate



Figure 6: mole fraction vs Stage number in rectifier block (Aspen Plus)

\section{Volume 6 Issue 4, April 2017 www.ijsr.net}




\section{International Journal of Science and Research (IJSR) \\ ISSN (Online): 2319-7064}

Index Copernicus Value (2015): 78.96 | Impact Factor (2015): 6.391

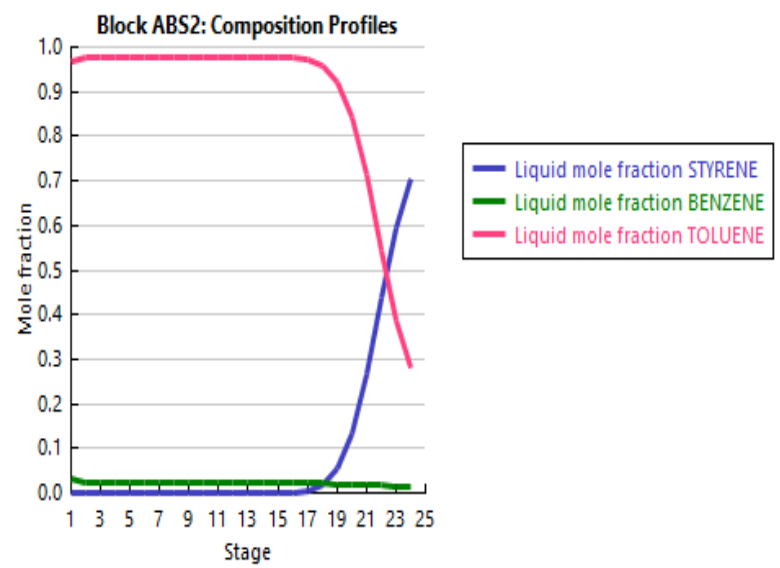

Figure 7: Mole fraction vs Stage number in stripper block (Aspen Plus)



Figure 8: mole fraction vs Stage number in absorber 2 block (Aspen Plus)

\section{Results}

Table 1: Comparision of Conventional and DWC system

\begin{tabular}{|c|c|c|}
\hline Parameters & Conventional system & DWC \\
\hline Number of Trays & 64 & 94 \\
\hline Energy Consumption(KW) & $2199.49 \mathrm{KW}$ & $1927.09 \mathrm{KW}$ \\
\hline Styrene Purity(\%) & $99.67 \%$ & $99.99 \%$ \\
\hline Toluene Purity(\%) & $76.15 \%$ & $96.00 \%$ \\
\hline Benzene Purity(\%) & $99.95 \%$ & $99.99 \%$ \\
\hline
\end{tabular}

It can be seen from the results table the Divided wall column not only saves energy but also gives us a better purity of toluene and styrene. The elimination of a reboiler and condenser in the DWC is the main reason behind the energy saving [8].

\section{Conclusion}

The Divided Wall Column system presents an advantage in comparison with the Conventional System as it not only reduced the energy consumption but also improved the purity of the final products. From the results obtained, it was observed that a considerable energy saving of approximately $272.4 \mathrm{KW}$ was obtained when using the DWC. The energy consumed by the Divided Wall Column was observed as 1927.09KW, while the energy consumption of the Conventional System was 2199.49 KW. The construction cost of the DWC is also less as compared to the conventional system as the use of one condenser and one reboiler is eliminated. Also in DWC the purity of Toluene increased from $76.15 \%$ to $96.00 \%$, while the purity of styrene increased from $99.67 \%$ to $99.99 \%$.

\section{References}

[1] M.Trung,Dung Nguyen, “Conceptual Design,Simulation and Experimental validaton of Divided wall column:Application for non-reactive and reactive mixture",Phd thesis,University of Toulouse, Toulouse, France,January-2015,p6-20,p95-120.

[2] Jonathan J. Parra Santiago,Carlos Alberto Guerrero Fajardo,Jose Ricardo Sodre, "Distillation process optimization for styrene production from a styrenebenzene-toluene system in a petlyuk column",Chemical engineering and Processing:Process Intensification,2015.

[3] Serra, M., Espuna, A. \& Puigjaner, L.,"Control and optimization of the divided wall column".Chemical Engineering and Processing,Vol.38,pp.549-562,1999.

[4] Kishore Khushalani, Akanksha Maheshwari, Nikita Jain, "Separation of Mixture by Divided Wall Column using Aspen Plus", International Journal of Emerging Technology and Advanced Engineering,, Issue 8,Volume 4,August 2014.

[5] Saurabh Arora (110CH0386), "Simulation Study of Divided Wall Distillation Column",Phd thesis, NationalInstituteofTechnology,Rourkela,India,p1828, 2013-2014.

[6] Vikas Kumar Sangal, L. Bichalu,Vineet Kumar and Indra Mani Mishra; "Importance of pressure drop in divided wall distillation column",Asia-Pacific Journal Of Chemical Engineering, Feb 2012.

[7] Nptel India, "Approximate methods for Multicomponent multistage

Distillation",[Online],Available:nptel.ac.in/courses/10310 7096/module5/lecture3/lecture3.pdf.

[8] Schultz, M.A., Stewart, D.G., Harris, J.M., Rosenblum, S.P., Shakur, M.S. and O`Brien, D.E.,Reduce Costs with Dividing Wall Columns, Chemical Engineering. Progress, pp.64-71,may 2002.

[9] R. Balasamy, A. Khurshid, A. Al-Ali, L. Atanda, K. Sagata, M. Asamoto, H. Yahiro,K. Nomura, T. Sano, K. Takehira, S. Al-Khattaf, Ethylbenzene dehydrogenation over binary $\mathrm{FeOx}-\mathrm{MeOy} / \mathrm{Mg}(\mathrm{Al}) \mathrm{O}$ catalysts derived from hydrotalcites: Role of $\mathrm{MgO}$ as basic sites, Appl Catal AGen, Vol.398,pp. 113-122, 2011.

\section{Volume 6 Issue 4, April 2017 www.ijsr.net}

